\title{
Energy Conversion by Molecular Motors Coupled to Nucleotide Hydrolysis
}

\author{
Reinhard Lipowsky • Steffen Liepelt • Angelo Valleriani
}

Received: 26 November 2008 / Accepted: 30 April 2009 / Published online: 13 May 2009

(c) The Author(s) 2009. This article is published with open access at Springerlink.com

\begin{abstract}
Recent theoretical work on the energy conversion by molecular motors coupled to nucleotide hydrolysis is reviewed. The most abundant nucleotide is provided by adenosine triphosphate (ATP) which is cleaved into adenosine diphosphate (ADP) and inorganic phosphate. The motors have several catalytic domains (or active sites), each of which can be empty or occupied by ATP or ADP. The chemical composition of all catalytic domains defines distinct nucleotide states of the motor which form a discrete state space. Each of these motor states is connected to several other states via chemical transitions. For stepping motors such as kinesin, which walk along cytoskeletal filaments, some motor states are also connected by mechanical transitions, during which the motor is displaced along the filament and able to perform mechanical work. The different motor states together with the possible chemical and mechanical transitions provide a network representation for the chemomechanical coupling of the motor molecule. The stochastic motor dynamics on these networks exhibits several distinct motor cycles, which represent the dominant pathways for different regimes of nucleotide concentrations and load force. For the kinesin motor, the competition of two such cycles determines the stall force, at which the motor velocity vanishes and the motor reverses its direction of motion. In general, kinesin is found to be governed by the competition of three distinct chemomechanical cycles. The corresponding network representation provides a unified description for all motor properties that have been determined by single molecule experiments.
\end{abstract}

Keywords Chemomechanical coupling $\cdot$ Molecular motor cycles $\cdot$ Energy balance relations $\cdot$ Entropy production $\cdot$ Stochastic motor dynamics

Dedicated to Edouard Brezin and Giorgio Parisi.

R. Lipowsky $(\bowtie) \cdot S$. Liepelt · A. Valleriani

Theory and Bio-Systems, Max-Planck-Institute of Colloids and Interfaces, 14424 Potsdam, Germany

e-mail: lipowsky@mpikg.mpg.de 


\section{Introduction}

Each cell of our body contains many different types of molecular motors and machines which fulfill different functions [1,2]. Most of these motors are powered by the hydrolysis of nucleotides such as adenosine triphosphate (ATP) or guanosine triphosphate (GTP). ATP is the most abundant of these molecules: the human body hydrolyzes and resynthesizes about $60 \mathrm{~kg}$ of ATP per day (or per marathon run). Thus, most motors represent ATPases, i.e., catalysts or enzymes for the hydrolysis of ATP. This hydrolysis process consists of several substeps: first, ATP is cleaved into ADP/P, i.e., bound adenosine diphosphate (ADP) and inorganic phosphate $(\mathrm{P})$, then the $\mathrm{P}$ is released from the motor, and finally the ADP is typically released as well.

For the concentrations which prevail in living cells, the ATP hydrolysis is strongly exergonic or 'downhill' but it is also quite slow in the absence of any enzymatic activity. The motors act as enzymes for this chemical reaction which leads to much faster hydrolysis rates. In addition, these motors are also able to transform the free energy released from the ATP hydrolysis into useful work. In fact, this free energy transduction occurs even at the level of single hydrolysis events. In this way, they consume quantized amounts of fuel.

Even though some of these motors have now been studied for more than a decade, both experimentally and theoretically, our understanding of their behavior is still rather limited. In particular, it has only recently been realized that these motors are governed by several motor cycles as explicitly shown for kinesin [3, 4]. The corresponding network models have revealed general balance conditions for the energy conversion by molecular motors that arise from thermodynamic constraints on the motor dynamics $[5,6]$.

Kinesin walks in a 'hand-over-hand' fashion, i.e., by alternating steps, in which one head moves forward while the other one remains bound to the filament [7, 8]. Each step corresponds to a motor displacement of $8 \mathrm{~nm}$ corresponding to the lattice constant of the microtubule. These mechanical steps of kinesin are fast and completed within $15 \mu$ s [9]. The kinesin motor exhibits tight coupling, i.e., it hydrolyzes one ATP molecule per mechanical step [10]. After ATP has been hydrolyzed by one of the catalytic motor domains (or active sites), the inorganic phosphate is released rather fast, and both transitions together take of the order of $10 \mathrm{~ms}$ to be completed [11]. ADP is subsequently released from the catalytic domain, and this release process is also completed during about $10 \mathrm{~ms}$ [12]. Thus, these chemical transitions take much longer than the mechanical steps. When the catalytic domain of one motor head is occupied by ADP, this head is only loosely bound to the microtubule [13-15] and most likely to unbind from it.

As shown in our recent theoretical studies, the kinesin motor is governed by three different cycles that dominate stepping dynamics depending on the ATP, ADP, and P concentrations as well as on the load force [3, 4]. The corresponding network of motor cycles provides a unified description for all motor properties that have been determined by single molecule experiments. For kinesin, the experimentally observed properties include motor velocity $[9,16,17]$, bound state diffusion coefficient (or randomness parameter) [16], ratio of forward to backward steps [9], dwell time distributions [9], and run length [18] as functions of ATP concentration and load force as well as motor velocity as a function of $\mathrm{P}$ and ADP concentration [19].

The directed stepping regime corresponds to the directed walks of the motor along the filament. Because of thermal noise, the motor makes, on average, a certain number of steps before it detaches from the filament. A single kinesin motor, for example, which moves under low load in the presence of plenty of ATP, unbinds from the filament after the motor has made about a hundred steps, i.e., after a run length (or walking distance) of about $1 \mu \mathrm{m}$ 
[20]. In general, the run length depends both on the nucleotide concentrations and on the load force, and our theory also provides a quantitative description for this dependence [3].

This short review is organized as follows. First, we address the chemomechanical coupling between ATP hydrolysis and mechanical steps and describe a systematic theoretical framework for this coupling. This framework incorporates the catalytic ATPase activity of the motor domains as well as basic thermodynamic constraints. When applied to kinesin, it leads to a unified description of all single molecule data that have been obtained for this motor. In addition, this framework also reveals that kinesin and other cytoskeletal motors are governed by several competing motor cycles.

\section{Enzymatic Activity of Motor Molecules}

For a given position at the filament, the molecular motor can attain many molecular conformations, which differ in the chemical composition of their catalytic domains and in thermally excited vibrational modes. Since we want to describe the hydrolysis of single ATP molecules, we will use a discrete state space and focus on the different chemical compositions of the catalytic domains. From a mathematical point of view, the chemical composition of the catalytic domains provides an equivalence relation that divides the molecular configurations of the motor into mutually distinct sets or equivalence classes.

We start with a single catalytic domain as shown in Fig. 1(a) and (b). Such a catalytic domain can be occupied by a single ATP molecule, by the combination ADP/P, by a single ADP molecule, or can be empty. In this way, each catalytic domain can attain 4 different nucleotide states, as shown in Fig. 1(a) where these states are represented as vertices in a network graph. Such a representation was previously used by T.L. Hill for a generic ATPase [21]. The edges between the different chemical states in Fig. 1(a) represent forward and backward transitions. The edge between state $i$ and state $j$ will be denoted by $\langle i j\rangle$. It consists of two directed edges or di-edges, $|i j\rangle$ and $|j i\rangle$, corresponding to the forward transition from $i$ to $j$ and to the backward transition from $j$ to $i$, respectively. Thus, the di-edge or transition $|\mathrm{ET}\rangle$ corresponds to ATP binding to the catalytic domain whereas the transition $|T E\rangle$ represents ATP release from this domain. Likewise, the transitions $|\Theta D\rangle,|D \Theta\rangle,|D E\rangle$, and $|E D\rangle$ describe $P$ release, $P$ binding, ADP release, and ADP binding, respectively. Finally, the transition $|\mathrm{T} \Theta\rangle$, corresponds to ATP cleavage and the transition $|\Theta \mathrm{T}\rangle$ to ATP synthesis from the ADP/P state.

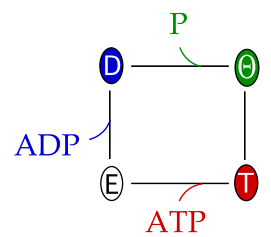

(a)

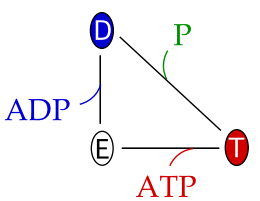

(b)

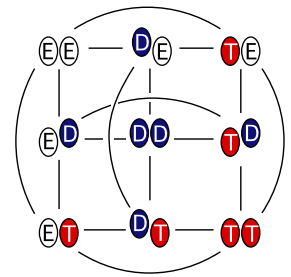

(c)

Fig. 1 Chemical networks of nucleotide states $(\mathbf{a}, \mathbf{b})$ for a single catalytic motor domain acting as an ATPase, and (c) for a motor with two catalytic motor domains. In (a), the catalytic motor domain can be empty (E), occupied by ATP $(\mathrm{T})$, by ADP/P $(\Theta)$, or by ADP (D). In (b), the cleavage transition $|\mathrm{T} \Theta\rangle$ and the P release transition $|\Theta D\rangle$ have been combined into the single transition $|\mathrm{TD}\rangle$ leading to a reduced network with the three states E, T, and D. In (c), the same three nucleotide states are accessible to each motor domain which implies a chemical network with $3^{2}=9$ states. Each solid line between two vertices $i$ and $j$ corresponds to both the forward chemical transition $|i j\rangle$ and the backward chemical transition $|j i\rangle$. Thus, the 9-state network in (c) contains 18 forward and 18 backward transitions 
The three edges $\langle\mathrm{ET}\rangle,\langle\Theta \mathrm{D}\rangle$, and $\langle\mathrm{DE}\rangle$ involve the binding and release of a certain molecular species from the aqueous solution. In contrast, the edge $\langle\mathrm{T} \Theta\rangle$ in the 4-state network does not involve such an interaction of the catalytic domain with the particle reservoir, see Fig. 1(a). Therefore, one may combine the two edges $\langle\mathrm{T} \Theta\rangle$ and $\langle\Theta \mathrm{D}\rangle$ of the 4-state network into the edge $\langle\mathrm{TD}\rangle$ as shown in Fig. 1(b). The latter representation involves only 3 nucleotide states: the motor head is occupied by ADP in state D, empty in state E, and occupied by ATP in state $\mathrm{T}$. This reduced representation can be defined in such a way that 3-state and 4-state network describe the same energy transduction process as shown in [6].

Next, we consider a two-headed motor such as kinesin or myosin V with two identical catalytic motor domains. If each motor domain can attain three different chemical states as in Fig. 1(b), the two-headed motor can attain $3^{2}=9$ different states as in Fig. 1(c) [3].

It is straightforward to generalize these considerations to a molecular motor with more than two catalytic domains. In general, one has to distinguish between the number $M_{\mathrm{bs}}$ of ATP binding sites and the number $M_{\mathrm{cd}}$ of catalytic domains for ATP hydrolysis with $M_{\mathrm{cd}} \leq M_{\mathrm{bs}}$. Dynein, for example, has two heads, each of which has four ATP binding sites, i.e., $M_{\mathrm{bs}}=8$, but only one or two of these four sites are believed to be catalytically active, i.e, $M_{\mathrm{cd}}=2$ or 4 . Likewise, the rotary motor F1 ATPase has $M_{\mathrm{bs}}=6$ and $M_{\mathrm{cd}}=3$ whereas the chaperone GroEL has $M_{\mathrm{bs}}=14$ and $M_{\mathrm{cd}}=7$.

For a motor with $M_{\mathrm{cd}}$ different catalytic domains, the different chemical compositions of the motor domains define $3^{M_{\mathrm{cd}}}$ nucleotide states of the motor, which are represented by $3^{M_{\mathrm{cd}}}$ vertices. Each of these vertices is connected to $2 M_{\mathrm{cd}}$ other vertices via chemical transitions, i.e., each vertex has 'chemical degree' $2 M_{\mathrm{cd}}$. As a result, one obtains a network with $M_{\mathrm{cd}} 3^{M_{\mathrm{cd}}}$ chemical edges, each of which represents both a forward and a backward chemical transition. This network represents an $M_{\mathrm{cd}}$-dimensional hypercube with periodic boundary conditions.

\section{Chemomechanical Coupling}

For cytoskeletal motors with two catalytic domains, we must complement the chemical network of nucleotide states as shown in Fig. 1(c) by mechanical transitions that represent the spatial displacements of the motors along the filaments. We will now use the convention that, for each state in Fig. 1(c), the right head is the leading head whereas the left head is the trailing head with respect to the prefered direction of the motor movement.

\subsection{Network Representations}

Both the kinesin motor [7, 8] and the myosin V motor [22] walk in a hand-over-hand fashion, i.e., by alternating steps, in which one head moves forward while the other one remains bound to the filament. In addition, recent experiments [9] provide strong evidence for the kinesin motor that the time scales for the mechanical and chemical transitions are well separated: the mechanical transitions are completed within a few microseconds whereas the chemical transitions take many milliseconds. If we incorporate these two properties into the network representation, we obtain nine possible mechanical transitions as shown in Fig. 2. For myosin V, the motor undergoes two [23] or three [24] mechanical substeps which implies that some of the chemical states are located at intermediate positions between the binding sites of the filament.

In principle, one may now construct a variety of chemomechanical networks by including different subsets of the possible mechanical transitions shown in Fig. 2(a)-(c). In general, this would lead to many possible pathways, a viewpoint that has been previously emphasized 
(a)

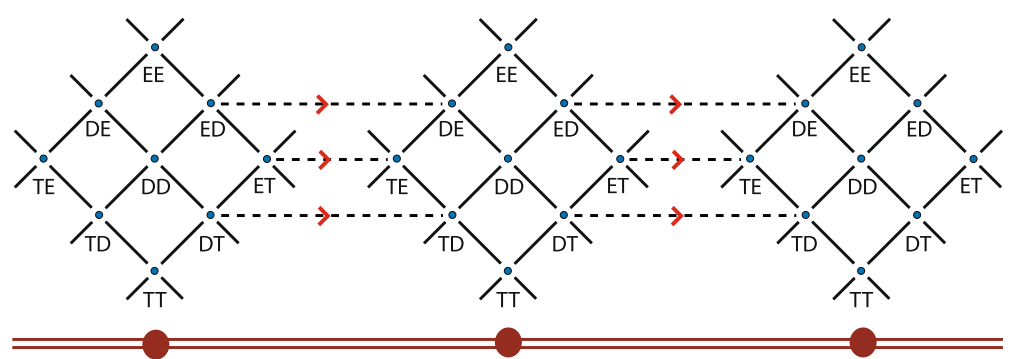

(b)

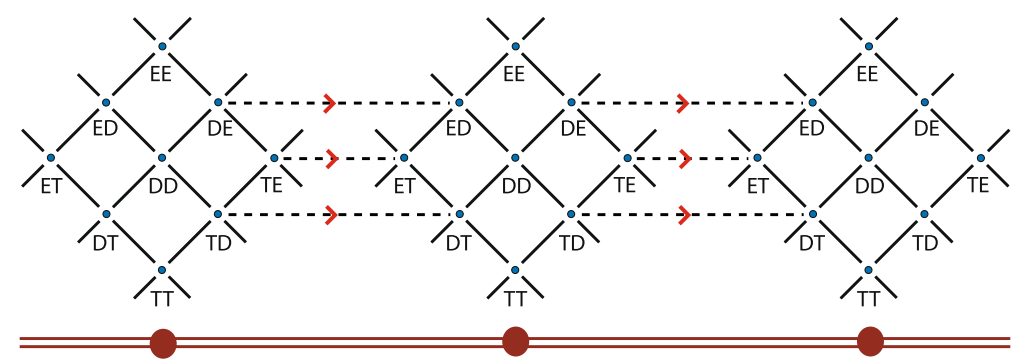

(c)

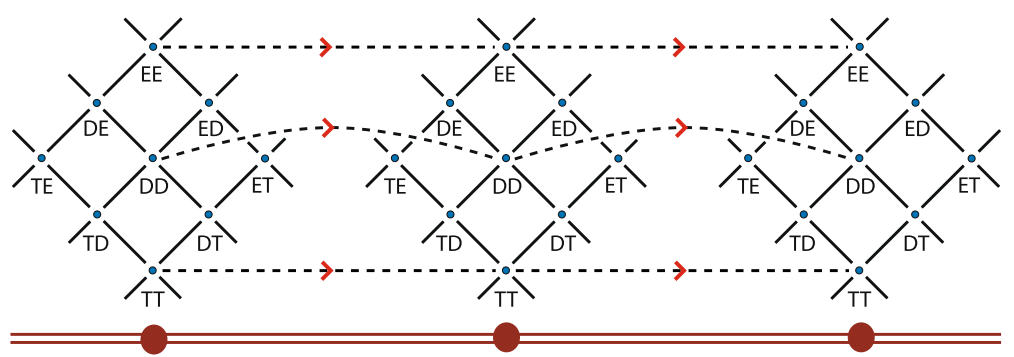

Fig. 2 (Color online) Possible mechanical transitions or steps between the chemical motor states at different binding sites of the filament. In principle, one has nine such transitions starting from the nine chemical motor states. For the sake of clarity, these nine possibilities have been divided up into three subgroups in subfigures (a), (b), and (c). The thick double-lines at the bottom of each subfigure represent the filament, the three bullets on these lines three filament binding sites. The motor can attain nine chemical states at each binding site. The solid and broken lines of the networks correspond to chemical and mechanical transitions, respectively. The arrows (red) indicate the forward direction of the mechanical transitions. The chemical networks have been drawn as square lattices with periodic boundary conditions; the stubs correspond to additional chemical transitions that connect the boundary states of these square lattices

for somewhat different network representations that did not include the chemical species ADP and P [25, 26]. In practise, the chemomechanical networks obtained from Fig. 2(a)-(c) can be simplified substantially as has been explicitly shown for kinesin [3].

For kinesin, the $\mathrm{T}$ and the $\mathrm{E}$ state of each motor head are strongly bound whereas the $\mathrm{D}$ state is only weakly bound to the filament [13-15]. In order to make a forward mechanical step, the trailing head must detach from the filament whereas the leading head must be firmly attached to it. It is then implausible that the motor starts its mechanical step from any state in which the trailing head is strongly bound and/or the leading head is weakly bound to the filament. This implies that the motor is unlikely to undergo this transition from the (ED), 


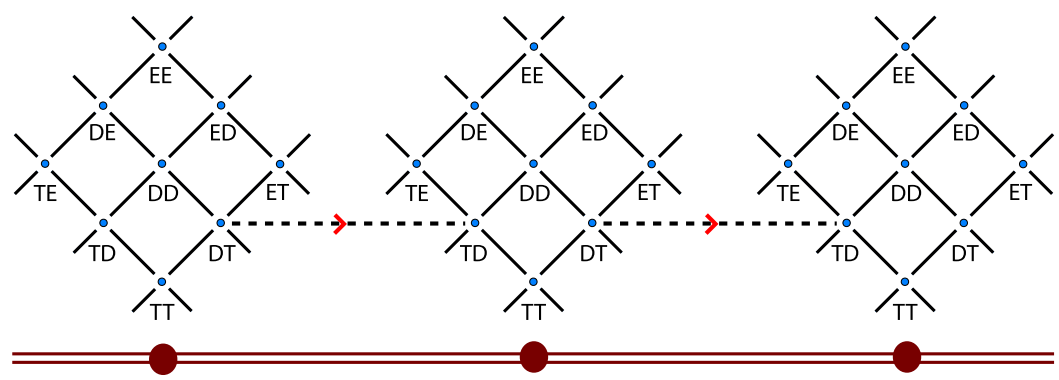

Fig. 3 Mechanical forward transition from state (DT) to state (TD) that should dominate for kinesin

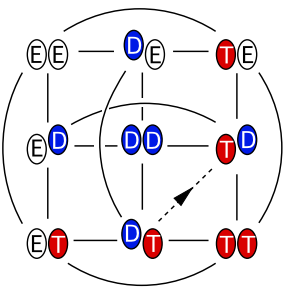

(a)

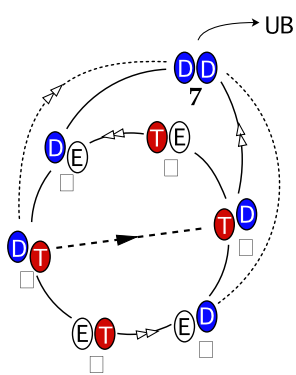

(b)

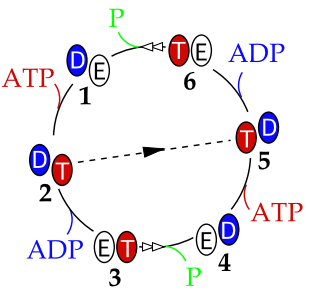

(c)

Fig. 4 Chemomechanical networks for the processive motion of kinesin with chemical (solid lines) and mechanical (broken lines) transitions: (a) Compact representation of the periodic network in Fig. 3; (b) Reduced 7-state network without the most strongly bound motor states (TT) and (EE); and (c) Reduced 6-state network without the most weakly bound motor state (DD). The white double arrows indicate the direction of ATP hydrolysis, the black arrows the direction of mechanical forward steps. The symbol UB in (b) represents the unbound state of the motor which can be reached from the (DD) state as will be discussed in Sect. 9 below [3]

(TD) or (DD) states as well as from the (ET), (TE), (EE), or (TT) states. One is then left with only two possible mechanical transitions in the forward direction, from state (DE) to state (ED) and from state (DT) to state (TD).

If the motor underwent its mechanical transition starting from the (DE) state, this mechanical transition would compete with the transition from (DE) to (DT). Since the transition rate for the latter transition increases with the ATP concentration, the frequency for the (DE) to (DT) transition would increase and the frequency for the (DE) to (ED) transition would decrease with increasing ATP concentration. Therefore, if the motor underwent its mechanical transition from (DE) to (ED), its velocity would decrease for high ATP concentration. Since such a decrease of motor velocity with ATP concentration is not observed experimentally, the mechanical forward transition of kinesin should correspond to the transition from the (DT) to the (TD) state. ${ }^{1}$ Thus, kinesin should be governed by the chemomechanical network in Fig. 3 as proposed in [3].

A more compact and equivalent representation for the kinesin network in Fig. 3 is shown in Fig. 4(a). Indeed, to each trajectory in the periodic network of Fig. 3, there is a cor-

\footnotetext{
${ }^{1}$ In principle, the motor could also alternate between several mechanical transitions such as the (DT) to (TD) transition and the (DE) to (ED) transition as shown in Fig. 2(a) and (b).
} 
responding trajectory in the compact network of Fig. 4(a) and vice versa. In addition, the spatial displacement along the filament can be recovered from the trajectories in the compact network of Fig. 4(a) by simply counting the number of forward and backward mechanical transitions from (DT) to (TD) and from (TD) to (DT), respectively.

The chemomechanical network as shown in Fig. 4(a) contains 36 chemical forward and backward transitions in addition to the forward and backward mechanical transitions. On the one hand, it is not possible at present to uniquely determine all of these rates from the available experimental data. On the other hand, it turns out that all of these data can be quantitatively described by the reduced 7-state network in Fig. 4(b) in which the two states (TT) and (EE) have been omitted. Indeed, one would expect that the motor avoids visiting these two states during its processive motion. As mentioned before, both the $\mathrm{T}$ and the $\mathrm{E}$ state of each motor head are strongly bound to the microtubule which implies that the (TT) and (EE) state are bound even more strongly to it. Thus, the motor would have to overcome relatively large energy barriers if it visited the (TT) or (EE) states during its processive motion. An even simpler description is obtained if one ignores the (DD) state as well which leads to the 6-state network in Fig. 4(c). The latter network is sufficient to describe all single motor data currently available apart from the strong reduction of the motor velocity with increasing ADP concentration [3].

\subsection{Cycles and Dicycles}

The previous subsections provided some examples for the description of molecular motors in terms of a discrete state space. These states are represented as the vertices of a network graph, $\mathbb{G}$, and are labeled by $i=1,2, \ldots,|\mathbb{G}|$. Two neighboring states $i$ and $j$ are connected by an edge $\langle i j\rangle$ which represents the two directed edges or transitions $|i j\rangle$ and $|j i\rangle$. Inspection of Fig. 4(a)-(c) shows that these edges form cycles. These cycles are particularly important in the present context since they are intimately related to fluxes and nonequilibrium steady states [21].

In order to be precise, we will distinguish (undirected) cycles from directed cycles or dicycles. The smallest dicycle consists of three states and three di-edges. An (undirected) cycle $\mathcal{C}_{v}$ is given by a closed sequence of neighboring vertices together with connecting edges, in which each vertex and each edge occurs only once. Each cycle $\mathcal{C}_{v}$ leads to two dicycles $\mathcal{C}_{v}^{d}$ with $d= \pm$.

The network description of a single motor head, see Fig. $1(a, b)$, involves only a single cycle. Analogous unicycle models have also been frequently used for two-headed motors. Inspection of Fig. 4 shows, however, that these two-headed motors will, in general, exhibit several motor cycles. The 9-state network in Fig. 4(a) involves a rather large number of cycles (more than 200). In contrast, the 6-state network in Fig. 4(c) contains only three cycles: the forward cycle $\mathcal{F}=\langle 25612\rangle$, the backward cycle $\mathcal{B}=\langle 52345\rangle$, and the dissipative slip cycle $\mathcal{D}=\langle 1234561\rangle$.

\section{Thermodynamics of Single Motors}

The chemomechanical pathways described in the previous section must satisfy some constraints arising from thermodynamics. Before we discuss these constraints in the next section, we will first describe an appropriate statistical ensemble for a single motor.

In order to do so, let us consider a single motor molecule bound to a filament that is embedded in a large amount of water at constant temperature $T$. During ATP hydrolysis, 


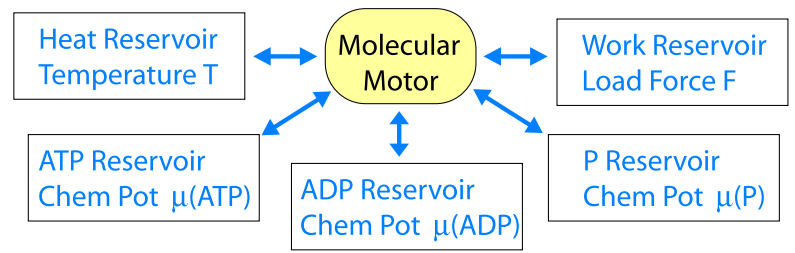

Fig. 5 Thermodynamic view of a molecular motor that is coupled to several reservoirs: a heat reservoir at temperature $T$; particle exchange reservoirs for ATP, ADP, and P with chemical potentials $\mu$ (ATP), $\mu$ (ADP), and $\mu(\mathrm{P})$; and a work reservoir governed by load force $F$. The motor is always taken to be in thermal equilibrium at temperature $T$ but can be in chemical equilibrium or nonequilibrium depending on the size of the three chemical potentials. Mechanical equilibrium corresponds to $F=0$ [6]

the motor binds ATP from the aqueous solution and releases ADP and P to it. In addition, the motor may experience a load force $F$ arising, e.g., from an optical trap. From the thermodynamic point of view, such a motor can be treated as a small system that is coupled to several reservoirs: (i) A heat reservoir at temperature $T$; (ii) A work reservoir characterized by the load force $F$; and (iii) Particle reservoirs for the chemical species $X=$ ATP, ADP, and inorganic phosphate P. These different types of reservoirs are displayed in Fig. 5.

The motor is taken to be in thermal equilibrium with its environment, i.e., the motor is characterized by the same temperature $T$ as the surrounding solution. The heat exchanged between the motor and the reservoir will be denoted by $Q$. We use the sign convention that the exchanged heat $Q$ is positive if it increases the internal energy of the reservoir. Thus, $Q>0$ corresponds to heat release from the motor to the reservoir.

We also use the conventions that the mechanical work $W_{\text {me }}$ is positive if the motor performs work on the work reservoir and that the exchanged chemical energy (or chemical work) $E_{\mathrm{ch}}$ is positive if the motor gains chemical energy from the particle exchange reservoirs. Using these sign conventions, the change $\Delta U$ of the motor's internal energy is written in the general form $[5,6]$

$$
\Delta U=E_{\mathrm{ch}}-W_{\mathrm{me}}-Q
$$

which represents the first law of thermodynamics. For chemical reaction networks without a work reservoir as recently considered in Refs. [27, 28], this relation reduces to $\Delta U=$ $E_{\text {ch }}-Q$.

Interaction of the molecular motor with the work reservoir is governed by the load force $F$. This force acts parallel to the filament and is taken to have a constant value independent of the spatial position of the motor. We use the sign convention that $F$ is positive if it acts against the prefered movement of the motor. If the motor moves by the distance $\ell$ in its prefered direction along the filament, it performs the mechanical work

$$
W_{\mathrm{me}}=\ell F>0 \text { for } F>0 \text {. }
$$

Mechanical equilibrium between motor and work reservoir corresponds to $F=0$.

In general, the external force as applied in single molecule experiments is a 3-dimensional vector with one component parallel to the filament defining the load force and additional components acting perpendicular to this filament. Since the motor does not perform mechanical work against the perpendicular force components, the latter components do not represent thermodynamic control parameters, even though they can affect the motor dynamics [6]. 
The exchanged chemical energy $E_{\mathrm{ch}}$ that contributes to the internal energy change (1) of the motor arises from adsorption and desorption processes between the motor and the surrounding aqueous solution. In the thermodynamic description used here, these processes are described by exchange equilibria between the motor and the reservoirs for the chemical species $X=$ ATP, ADP, and P, see Fig. 5. It is important to note that we consider three separate reservoirs that are not in chemical equilibrium with each other. Chemical equilibrium between the three particle exchange reservoirs would arise from ATP hydrolysis and synthesis in the absence of the motor molecule. The latter processes are, however, very slow, because they have to overcome a large energy barrier, and, thus, can be ignored on the relevant time scales.

The exchange equilibria between the motor and the reservoirs for the chemical species $X=\mathrm{ATP}, \mathrm{ADP}$, and $\mathrm{P}$ are governed by the corresponding chemical potentials, $\mu(X)$. The activity of $X$ will be denoted by $[X]$ and is equal to the molar concentration in the limit of dilute solutions. In the following, we will use the term 'concentration' to be a synonym for 'activity'. For each activity $[X]$, we choose the activity scale $[X]^{*}$ in such a way that the chemical potential $\mu(X)$ has the simple form

$$
\mu(X)=k_{B} T \ln \left([X] /[X]^{*}\right)
$$

with the Boltzmann constant $k_{B}{ }^{2}$

When the motor hydrolyzes a single ATP molecule, it binds one such molecule from the ATP reservoir and releases one inorganic phosphate $\mathrm{P}$ and one ADP molecule into the ADP and $\mathrm{P}$ reservoirs, compare Fig. 5. The corresponding chemical energy $E_{\mathrm{ch}}$ for one hydrolysis process is then given by

$$
E_{\mathrm{ch}}=\mu(\mathrm{ATP})-\mu(\mathrm{P})-\mu(\mathrm{ADP}) \equiv \Delta \mu
$$

which also represents the chemical energy input from the aqueous solution to the motor molecule. Inserting the expression (3) for the three chemical potentials into (4), we obtain

$$
\Delta \mu=k_{B} T \ln \left(\frac{[\mathrm{ATP}]}{[\mathrm{ADP}][\mathrm{P}]} K^{\mathrm{eq}}\right) \quad \text { with } K^{\mathrm{eq}} \equiv \frac{[\mathrm{ADP}]^{*}[\mathrm{P}]^{*}}{[\mathrm{ATP}]^{*}}
$$

which defines the equilibrium (dissociation) constant $K^{\text {eq }}$. Chemical equilibrium between ATP hydrolysis and ATP synthesis corresponds to $\Delta \mu=0$ which implies

$$
K^{\mathrm{eq}}=\left.\frac{[\mathrm{ADP}][\mathrm{P}]}{[\mathrm{ATP}]}\right|^{\mathrm{eq}} \text {. }
$$

For dilute solutions, the activities of the three chemical species are equal to their molar concentrations and can be directly measured, at least in principle (after the system has relaxed into chemical equilibrium). For ATP hydrolysis, the precise value of the equilibrium constant $K^{\text {eq }}$ depends on the ionic conditions but a typical value is given by $K^{\text {eq }}=4.9 \times 10^{11} \mu \mathrm{M}[19,29]$. Thus, in thermal equilibrium at temperature $T$, a single

\footnotetext{
${ }^{2}$ In general, the activity $[X]$ is defined in such a way that the chemical potential $\mu_{X}$ for the chemical species $X$ has the simple form $\mu_{X} \equiv \mu_{X}^{o}+k_{B} T \ln \left([X] /[X]^{o}\right)$ where the superscript $o$ refers to some standard or reference activity $[X]^{o}$. This relation can be rewritten in the form $[X]^{o} e^{-\mu_{X}^{o} / k_{B} T}=[X] e^{-\mu_{X} / k_{B} T}$ which holds for any reference state. Therefore, the right hand side of this equation can be used to define the activity scale $[X]^{*} \equiv[X] e^{-\mu_{X} / k_{B} T}$ which has a unique value independent of the reference activity $[X]^{o}$.
} 
molecular motor is governed by four thermodynamic control parameters, namely the three activities or concentrations $[X]$ with $X=$ ATP, ADP, and $\mathrm{P}$ as well as the load force $F$ or, equivalently, the three chemical potentials $\mu(X)$ and $F$.

Since the chemical energy input $\Delta \mu$ as given by (5) depends on all three concentrations [ATP], [ADP], and [P], the limit, in which one of these concentrations becomes small, is not defined unless one specifies the two remaining concentrations as well. If one considers the limit of small [ATP] for fixed [ADP] and [P], for example, the chemical energy input $\Delta \mu$ goes to minus infinity. Likewise, if one considers the limit of small [ADP] and/or small [P] for fixed [ATP], the energy input $\Delta \mu$ goes to plus infinity. Furthermore, if all three concentrations become small with $[\mathrm{ATP}] \approx[\mathrm{ADP}][\mathrm{P}] / K$ with a certain, fixed activity or concentration $K, \Delta \mu$ attains the limiting value $\Delta \mu=\ln \left(K^{\mathrm{eq}} / K\right)$. Thus, even if all three concentrations vanish simultaneously, the limiting value of $\Delta \mu$ depends on how they vanish.

In the absence of ATP, ADP, and P, the motor does not obtain any chemical energy and, thus, cannot perform any mechanical work. Thus, one would like to view this situation as a limiting case of chemical equilibrium with $\Delta \mu=0$. The latter value is obtained if all three concentrations vanish simultaneously with the constraint that $[\mathrm{ATP}] \approx[\mathrm{ADP}][\mathrm{P}] / K^{\mathrm{eq}}$.

\section{Energy Balance Conditions}

We now return to the network representation of the molecular motor as shown for kinesin in Fig. 4. As explained in the Appendix, each state of the motor represents an ensemble of substates that all have the same chemical composition and the same position along the filament but represent different molecular conformations [6]. These substates are taken to be equilibrated at temperature $T$, the usual assumption in classical transition-rate theory and Kramers theory for chemical kinetics, see references in [6]. As briefly reviewed in the Appendix, each nucleotide state $i$ can then be characterized by its internal energy $U_{i}$. During the transition $|i j\rangle$ from state $i$ to state $j$, the motor gains the chemical energy $E_{\mathrm{ch}, i j}$, performs the work $W_{\mathrm{me}, i j}$, and releases the heat $Q_{i j}$ into the heat reservoir. Therefore, the change in the internal energy during the transition $|i j\rangle$ is given by $[5,6]$

$$
U_{j}-U_{i}=E_{\mathrm{ch}, i j}-W_{\mathrm{me}, i j}-Q_{i j} .
$$

This relation is obtained when the first law of thermodynamics as described by (1) is applied to transitions between the two discrete states $i$ and $j$.

Starting from the local energy balance relations (7), we can derive nonlocal balance relations that are associated with the dicycles $\mathcal{C}_{v}^{d}$ of the network, see Sect. 3.2, and do not depend on the state variables $U_{i}$. Indeed, when we sum the relation (7) over all transitions $|i j\rangle$ of a given dicycle $\mathcal{C}_{v}^{d}$, the internal energies $U_{i}$ cancel out and we obtain

$$
\sum_{|i j\rangle}^{v, d}\left(U_{j}-U_{i}\right)=0=E_{\mathrm{ch}}\left(\mathcal{C}_{v}^{d}\right)-W_{\mathrm{me}}\left(\mathcal{C}_{v}^{d}\right)-Q\left(\mathcal{C}_{v}^{d}\right)
$$

with the exchanged chemical energy

$$
E_{\mathrm{ch}}\left(\mathcal{C}_{v}^{d}\right) \equiv \sum_{|i j\rangle}^{v, d} E_{\mathrm{ch}, i j}
$$

the average mechanical work

$$
W_{\mathrm{me}}\left(\mathcal{C}_{v}^{d}\right) \equiv \sum_{|i j\rangle}^{v, d} W_{\mathrm{me}, i j}
$$


and the average heat

$$
Q\left(\mathcal{C}_{v}^{d}\right) \equiv \sum_{|i j\rangle}^{v, d} Q_{i j},
$$

where all of these quantities now refer to one completion of the dicycle $\mathcal{C}_{v}^{d}$.

In general, the three terms (9)-(11) may have either sign or may vanish in special cases. By definition, the chemical energy $E_{\mathrm{ch}}\left(\mathcal{C}_{v}^{d}\right)>0$ if the motor gains chemical energy during the dicycle $\mathcal{C}_{v}^{d}$, the mechanical work $W_{\text {me }}\left(\mathcal{C}_{v}^{d}\right)>0$ if the motor performs work on the work reservoir, and the exchanged heat $Q\left(\mathcal{C}_{v}^{d}\right)>0$ if the motor releases heat into the heat reservoir. Furthermore, all of these terms change sign if one reverses the direction $d$ of the dicycle, i.e., $E_{\mathrm{ch}}\left(\mathcal{C}_{v}^{-d}\right)=-E_{\mathrm{ch}}\left(\mathcal{C}_{v}^{d}\right), W_{\text {me }}\left(\mathcal{C}_{v}^{-d}\right)=-W_{\text {me }}\left(\mathcal{C}_{v}^{d}\right)$, and $Q\left(\mathcal{C}_{v}^{-d}\right)=-Q\left(\mathcal{C}_{v}^{d}\right)$. A special case is provided by a single catalytic motor domain as depicted in Fig. 1(a) and (b), which is characterized by a single cycle, say $C_{1}$. For such a single domain that does not perform mechanical work, one has $W_{\text {me }}\left(\mathcal{C}_{1}^{d}\right)=0$, and the chemical energy input $E_{\text {ch }}\left(\mathcal{C}_{1}^{+}\right)$is completely dissipated into heat [6].

In order to obtain a quantitative model for the motor dynamics, one has to specify the transition rates $\omega_{i j}$ for transitions $|i j\rangle$ between the motor states $i$ and $j$. As shown in [5, 6], the released heat $Q\left(\mathcal{C}_{v}^{d}\right)$ is related to the transition rates $\omega_{i j}$ by

$$
Q\left(\mathcal{C}_{v}^{d}\right) \equiv k_{B} T \bar{Q}\left(\mathcal{C}_{v}^{d}\right)=k_{B} T \ln \left(\frac{\Pi_{\omega}\left(\mathcal{C}_{v}^{d}\right)}{\Pi_{\omega}\left(\mathcal{C}_{v}^{-d}\right)}\right)
$$

with the transition rate products

$$
\Pi_{\omega}\left(\mathcal{C}_{\nu}^{d}\right) \equiv \prod_{|i j\rangle}^{v, d} \omega_{i j}
$$

where the product includes all directed edges or transitions of the dicycle $\mathcal{C}_{v}^{d}$. The dicycle relation (12) was originally derived from the decomposition of the entropy production rate into dicycle contributions [5] but may also be derived from the entropy $k_{B} T \ln \left(\omega_{i j} / \omega_{j i}\right)$ produced during a single transition [30], see the Appendix. The relation (12) can be rewritten in a form that is reminiscent of the various fluctuation theorems for entropy fluctuations [31-34] as explained in [6].

The relation (12) was first derived in [5, 6] for Markov processes which are characterized by an exponential distribution for the dwell times $\tau_{i}$ of the motor states $i$. It turns out that the same relation is also valid for continuous-time random walks in which the motor is governed, at each state $i$, by transition probabilities $\pi_{i j}$ with $\sum_{j} \pi_{i j}=1$ and a nonexponential distribution $\psi_{i}\left(\tau_{i}\right)$ provided this distribution has a finite first moment, $\left\langle\tau_{i}\right\rangle=\int_{0}^{\infty} d \tau_{i} \psi_{i}\left(\tau_{i}\right) \tau_{i}<\infty$ for all motor states $i$. The transition rates $\omega_{i j}$ in (13) are then replaced by the effective transition rates $\hat{\omega}_{i j} \equiv \pi_{i j} /\left\langle\tau_{i}\right\rangle$.

A combination of the relations (8) and (12) then leads to the dicycle balance conditions $[5,6]$

$$
k_{B} T \ln \left(\frac{\Pi_{\omega}\left(\mathcal{C}_{v}^{d}\right)}{\Pi_{\omega}\left(\mathcal{C}_{v}^{-d}\right)}\right)=E_{\mathrm{ch}}\left(\mathcal{C}_{v}^{d}\right)-W_{\mathrm{me}}\left(\mathcal{C}_{v}^{d}\right)
$$

for the transition rates $\omega_{i j}$. Indeed, the chemical energy $E_{\mathrm{ch}}\left(\mathcal{C}_{v}^{d}\right)$ can be expressed in terms of the chemical energy difference $\Delta \mu$ obtained from the hydrolysis of a single ATP molecule as given by (4), and the mechanical work $W_{\text {me }}\left(\mathcal{C}_{v}^{d}\right)$ depends on the load force $F$ and on the step size $\ell$, compare (2). Therefore, the balance conditions (14) represent thermodynamic 
constraints on the kinetics. Note that the balance conditions for $\mathcal{C}_{v}^{+}$and $\mathcal{C}_{v}^{-}$differ only by an overall sign and are, thus, linearly dependent. In fact, the number of linearly independent balance conditions is equal to the number of fundamental cycles of the network.

\section{Motor Dynamics for Processive Stepping}

For the 6-state network of kinesin as displayed in Fig. 4(c), one has three cycles and two fundamental ones; for the 7-state network in Fig. 4(b) without the two dotted edges, one has six cycles and three fundamental ones. When these networks are supplemented by the associated balance conditions, one obtains a rather good description for all properties of the kinesin motor as experimentally observed in single motor experiments [3]. In these experiments, the average motor velocity $v$ has been measured as a function of the ATP, ADP, and P concentrations as well as of the load force $F$. The latter dependence is shown in Fig. 6(a) where the experimental data of Carter and Cross [9] are compared with the result of the network calculations as obtained in [3]. Inspection of Fig. 6(a) shows very good agreement between theory and experiment. The same agreement is found for the ratio $q$ of forward to backward steps as a function of load force $F$ as shown in Fig. 6(b). The network calculations also provide very good descriptions for the experimental data in Refs. [16-19].

Another important consequence of the balance conditions (14) is that they determine the general form of the dicycle excess fluxes in the steady state. The latter fluxes are defined via $[5,6]$

$$
\Delta J^{\mathrm{st}}\left(\mathcal{C}_{v}^{d}\right) \equiv J^{\mathrm{st}}\left(\mathcal{C}_{v}^{d}\right)-J^{\mathrm{st}}\left(\mathcal{C}_{v}^{-d}\right),
$$

i.e., by the difference of the steady state dicycle fluxes $J^{\text {st }}\left(\mathcal{C}_{v}^{d}\right)$ with $d= \pm$. The dicycle fluxes have the general properties

$$
J^{\mathrm{st}}\left(\mathcal{C}_{v}^{d}\right)>0 \quad \text { and } \quad J^{\mathrm{st}}\left(\mathcal{C}_{v}^{d}\right) \propto \Pi_{\omega}\left(\mathcal{C}_{v}^{d}\right)
$$

with a proportionality factor that depends only on the cycle $\mathcal{C}_{v}$ but not on its direction $d= \pm$ as can be explicitly shown using the graph-theoretic or diagrammatic solution for the steady state probabilities (often referred to as the Kirchhoff method). Using the relation between the transition rate products $\Pi_{\omega}$ and the released heat $Q\left(\mathcal{C}_{v}^{d}\right)$ as given by (12), one concludes
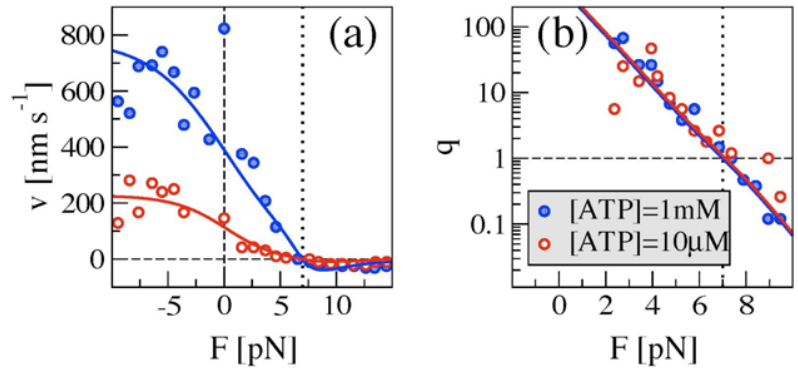

Fig. 6 (a) Average motor velocity $v$ and (b) forward to backward step ratio $q$ for the kinesin motor as a function of load force $F$ for two different values of the ATP concentration. The experimental data are from [9]. The solid lines are calculated using the chemomechanical networks in Figs. 4(b) and 4(c) [3]. At the stall force $F=F_{\mathrm{S}} \simeq 7 \mathrm{pN}$ indicated by the vertical dotted line, the velocity $v$ vanishes and the step ratio $q=1$ 
that the dicycle excess fluxes have the generic form

$$
\Delta J^{\mathrm{st}}\left(\mathcal{C}_{v}^{d}\right)=\left[1-e^{-\bar{Q}\left(\mathcal{C}_{v}^{d}\right)}\right] J^{\mathrm{st}}\left(\mathcal{C}_{v}^{d}\right)
$$

Since the flux $J^{\text {st }}\left(\mathcal{C}_{v}^{d}\right)$ is always positive, the expression (17) for the dicycle excess flux implies that

$$
\text { sign of } \Delta J^{\mathrm{st}}\left(\mathcal{C}_{v}^{d}\right)=\operatorname{sign} \text { of } \bar{Q}\left(\mathcal{C}_{v}^{d}\right)
$$

and that

$$
\Delta J^{\mathrm{st}}\left(\mathcal{C}_{v}^{d}\right)=0 \quad \text { if and only if } \quad \bar{Q}\left(\mathcal{C}_{v}^{d}\right)=0,
$$

i.e., the dicycle excess flux $\Delta J^{\text {st }}\left(\mathcal{C}_{v}^{d}\right)$ vanishes for those values of the thermodynamic control parameters for which the released heat $\bar{Q}\left(\mathcal{C}_{v}^{d}\right)$ vanishes.

Since the released heat $\bar{Q}$ is a thermodynamic quantity, thermodynamics alone determines the sign and the zeros of all dicycle excess fluxes $\Delta J^{\text {st }}\left(\mathcal{C}_{v}^{d}\right)$ in the steady state. Many experimentally accessible quantities such as the motor velocity and the ATP hydrolysis rate correspond to linear combinations of the dicycle excess fluxes. In fact, using the above mentioned properties of the dicycle excess fluxes both for the motor velocity and the ATP hydrolysis rate, one can identify four different operation modes for kinesin [35].

\section{Motor Velocity and Stall Force}

The force-velocity relationship as shown in Fig. 6(a) for kinesin represents an important property of all stepping motors. Inspection of Fig. 6(a) shows that the motor velocity decreases with increasing load force $F$ and vanishes at a characteristic force scale, the stall force $F=F_{\mathrm{s}}$. Intuitively, one may view this stall force as the maximal force that can be generated by the motor.

For the kinesin networks in Figs. 4(b) and 4(c), the parameter dependence of this stall force can be determined explicitly. In these networks, the motor velocity is proportional to the excess flux $\Delta J_{25}^{\text {st }}=P_{2}^{\text {st }} \omega_{25}-P_{5}^{\text {st }} \omega_{52}$ from state 2 to state 5 with the steady state probabilities $P_{2}^{\text {st }}$ and $P_{5}^{\text {st }}$ to find the motor in state 2 and 5, respectively. For the 6-state network in Fig. 4(c), one then has [3]

$$
v=\ell \Delta J_{25}^{\mathrm{st}}=\ell\left[\Delta J^{\mathrm{st}}\left(\mathcal{F}^{+}\right)-\Delta J^{\mathrm{st}}\left(\mathcal{B}^{+}\right)\right]
$$

with the step size $\ell$ and the two dicycles $\mathcal{F}^{+}=|25612\rangle$ and $\mathcal{B}^{+}=|23452\rangle$. For the transition rates as chosen in [3], the motor velocity then vanishes at the rescaled stall force

$$
\bar{F}_{\mathrm{s}} \equiv \frac{\ell F_{\mathrm{s}}}{k_{B} T}=\ln \left(\frac{e^{\bar{F}_{\infty}}+e^{-\Delta \bar{\mu}}}{1+e^{\bar{F}_{\infty}-\Delta \bar{\mu}}}\right)
$$

with $\Delta \bar{\mu} \equiv \Delta \mu / k_{B} T$ and $\bar{F}_{\infty} \equiv \ln \left[\omega_{25}(F=0) / \omega_{52}(F=0)\right]$. The relation (21) leads to the asymptotic behavior

$$
\bar{F}_{\mathrm{s}} \approx \bar{F}_{\infty} \text { for large } \Delta \bar{\mu}
$$

and

$$
\bar{F}_{\mathrm{s}} \approx \frac{e^{\bar{F}_{\infty}}-1}{e^{\bar{F}_{\infty}}+1} \Delta \bar{\mu} \quad \text { for small } \Delta \bar{\mu}
$$




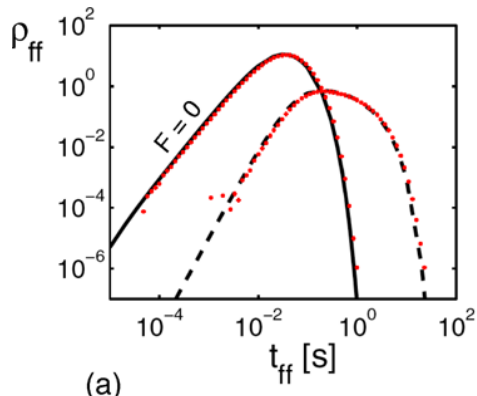

(a)

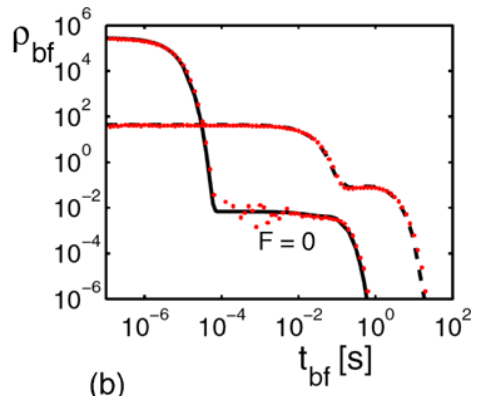

(b)

Fig. 7 (a) Probability distribution $\rho_{\mathrm{ff}}$ for the dwell time $t_{\mathrm{ff}}$ of mechanical forward-after-forward steps, i.e., the dwell time between two successive forward steps; and (b) Probability distribution $\rho_{\text {bf }}$ for the dwell time $t_{\mathrm{bf}}$ of mechanical forward-after-backward steps. In both cases, one curve corresponds to vanishing load force $F=0$ (as indicated), the other to $F=F_{\mathrm{s}}$, i.e., the load force being equal to the stall force. In addition, the motor dynamics leads to the probability densities $\rho_{\mathrm{fb}}$ and $\rho_{\mathrm{bb}}$ for backward-after-forward steps and backward-after-backward steps, respectively [4]

Since $k_{B} T / \ell=0.5 \mathrm{pN}$ at room temperature, the value $F_{\infty} \simeq 7 \mathrm{pN}$ as determined experimentally $[9,16]$ implies $\bar{F}_{\infty} \simeq 14$. An explicit expression for the stall force can also be obtained for the 7-state network in Fig. 4(b). In fact, analyzing both the motor velocity and the ATP hydrolysis rate, one can identify four different operation modes for kinesin [35].

\section{Dwell Time Distributions for Mechanical Steps}

In single motor experiments using optical traps, one can observe the spatial position of the motor as a function of time. For low ATP concentrations, the motor dwells in a certain position along the filament and, then, makes a fast mechanical transition to a new spatial position. If one measures many dwell times between successive mechanical transitions, one obtains the dwell time distributions of the motor.

As pointed out in [4], there are, in fact, four different dwell time distributions corresponding to the four possible pairs of subsequent forward and backward steps, namely forward-after-forward steps, forward-after-backward steps, backward-after-forward steps, and backward-after-backward steps. For kinesin, these dwell time distributions have been calculated starting from the 6-state network in Fig. 4(c) [4]. Two of these four distributions are displayed in Fig. 7. Inspection of this figure shows that these distributions have a strongly non-exponential character reflecting the underlying network dynamics involving chemical transitions between the different motor states.

The dwell time distributions for kinesin can be calculated from the extended network in Fig. 8 which is obtained from the 6-state network in Fig. 4(c) by the addition of the two absorbing states $j=2^{\prime}$ and $j=5^{\prime}$. On this extended network, the motor starts in the initial state $i=5$ after a forward step or in the initial state $i=2$ after a backward step. One then considers the probability $P_{i j}(t)$ that the motor is 'absorbed' after time $t$ in state $j=5^{\prime}$ corresponding to a final forward step or in state $j=2^{\prime}$ corresponding to a final backward step.

The corresponding absorption times $t_{i \mid j}^{\mathrm{ab}}$ are governed by the probabilities [4]

$$
\operatorname{Pr}\left\{t_{i \mid j}^{\mathrm{ab}} \leq t\right\}=\int_{0}^{t} d u \rho_{i \mid j}^{\mathrm{ab}}(u)
$$




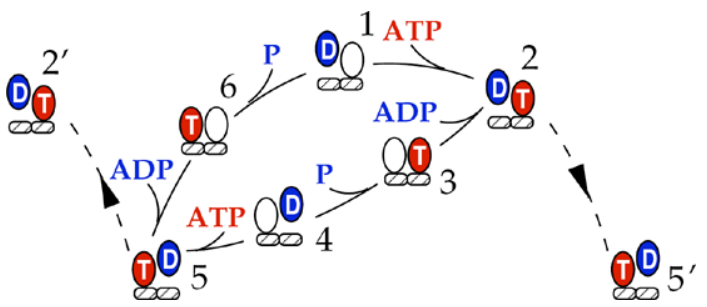

Fig. 8 Extended (6+2)-state network for kinesin as obtained from the 6-state network in Fig. 4(c) by adding the two adsorbing states $j=2^{\prime}$ and $j=5^{\prime}$. The motor undergoes a forward-after-forward step if it starts initially in state $i=5$ and is subsequently 'absorbed' in state $j=5^{\prime}$. Likewise, the motor undergoes a forward-after-backward step if it starts initially in state $i=2$ and is subsequently 'absorbed' in state $j=5$ '

with the probability distribution ${ }^{3}$

$$
\rho_{i \mid j}^{\mathrm{ab}}(t)=\frac{\partial}{\partial t} P_{i j}(t) / P_{i j}^{\mathrm{st}}
$$

where $P_{i j}^{\text {st }}$ is the steady state solution for the probability $P_{i j}(t)$. Thus, the two probability distributions $\rho_{\mathrm{ff}}$ and $\rho_{\mathrm{bf}}$ as displayed in Fig. 7 are obtained from the relations

$$
\rho_{\mathrm{ff}}(t)=\frac{\partial}{\partial t} P_{55^{\prime}}(t) / P_{55^{\prime}}^{\mathrm{st}} \quad \text { and } \quad \rho_{\mathrm{bf}}(t)=\frac{\partial}{\partial t} P_{25^{\prime}}(t) / P_{25^{\prime}}^{\mathrm{st}}
$$

the two remaining dwell time distributions $\rho_{\mathrm{fb}}$ and $\rho_{\mathrm{bb}}$ for backward-after-forward steps and backward-after-backward steps can be calculated in an analogous fashion [4].

Linear combinations of the four dwell time distributions $\rho_{\mathrm{ff}}, \rho_{\mathrm{bf}}, \rho_{\mathrm{fb}}$, and $\rho_{\mathrm{bb}}$ determine the probability distributions for forward and backward steps [4]. For kinesin, the latter distributions have been determined experimentally [9]. More precisely, these distributions have been measured for dwell times that exceed a certain small time cutoff that varies between 0.01 and $0.1 \mathrm{~s}$ depending on ATP concentration and load force. As shown in [4], these experimental data are very well described by the theoretical distributions as calculated for the 6-state network of kinesin. This agreement is quite remarkable since all transition rates used in this calculation have been obtained in [3] without any reference to the dwell time distributions. Thus, the agreement between theory and experiment is obtained without any additional fitting parameter.

\section{Unbinding Rate and Run Length}

The processive motors considered here can make many successive steps corresponding to many successive motor cycles. However, even if the motor's binding energy is large compared to the thermal energy $k_{B} T$, thermal fluctuations will eventually lead to an unbinding of the motor from the filament. Thus, a single motor can be characterized by its average run time $\langle\Delta t\rangle$ and the corresponding unbinding rate

$$
\omega_{\text {off }} \equiv 1 /\langle\Delta t\rangle
$$

\footnotetext{
${ }^{3}$ In mathematics, the quantity $\rho_{i \mid j}^{\mathrm{ab}}(t)$ is called a probability density function.
} 
During its run time, the single motor steps along the filament and covers the average run length

$$
\langle\Delta x\rangle=v\langle\Delta t\rangle .
$$

In the absence of load, kinesin motors bound to microtubules make about 100 successive steps [20] which corresponds to an average run length $\langle\Delta x\rangle \simeq 1 \mu \mathrm{m}$ and an average run time $\langle\Delta t\rangle \simeq 1 \mathrm{~s}$. In the absence and presence of dynactin, an accessory protein, dynein motors make about 20 and 40 successive steps, respectively [36]. Myosin V, which moves along actin filaments, makes about 50 steps before it unbinds again corresponding to a run length of about $1.5 \mu \mathrm{m}$ [37]. Myosin VI, on the other hand, makes only about 9 successive steps, i.e., its average run length is about $280 \mathrm{~nm}$ [38].

For a single motor stepping along a uniform filament as considered here, the motor's run time is equal to the motor's binding time, i.e., the time during which the motor is bound to the filament. In general, these two time scales can be different. Two examples are provided by a single motor that encounters obstacles on the filament [39] or by a cargo particle that is pulled by two antagonistic motor teams [40]. In these latter cases, the run times are shorter than the binding times.

The unbinding of a single motor is an activated process governed by a corresponding energy barrier. In the presence of an external force $F$ that acts to detach the motor, this barrier is reduced by $F \ell_{\mathrm{d}}$ where $\ell_{\mathrm{d}}$ represents an appropriate molecular length scale, which characterizes the elastic deformation of the motor molecule required for its detachment. Therefore, the unbinding rate $\omega_{\text {off }}$ is expected to have the general form

$$
\omega_{\text {off }}(F)=\kappa_{\text {off }} \exp \left(\ell_{\mathrm{d}} F / k_{B} T\right) \equiv \kappa_{\text {off }} \exp \left(F / F_{\mathrm{d}}\right)
$$

as follows from Kramers theory for activated processes with the zero-force unbinding rate $\kappa_{\text {off }}$ and the detachment force

$$
F_{\mathrm{d}} \equiv k_{B} T / \ell_{\mathrm{d}}
$$

Now, let us again focus on the network representations for kinesin as displayed in Fig. 4. In order to describe the unbinding of the motor from the filament, we must extend these networks by unbound motor states as indicated by 'UB' in Fig. 4(b). It is convenient to label these unbound states by $i=0$. Thus, if the motor dwells in a state $i>0$, its unbinding rate $\omega_{i 0}$ should have the load dependent form

$$
\omega_{i 0}=\kappa_{i 0} \exp \left(\ell_{\mathrm{d}, i} F / k_{B} T\right) \equiv \kappa_{i 0} \exp \left(F / F_{\mathrm{d}, i}\right) \equiv \kappa_{i 0} \exp \left(\chi_{i 0} \bar{F}\right)
$$

which defines the zero-force unbinding rates $\kappa_{i 0}$, the detachment forces $F_{\mathrm{d}, i} \equiv k_{B} T / \ell_{\mathrm{d}, i}$, the dimensionless parameters $\chi_{i 0} \equiv \ell_{\mathrm{d}, i} / \ell$, and the dimensionless force $\bar{F} \equiv \ell F / k_{B} T$, compare (21). Intuitively, one may view the detachment force $F_{\mathrm{d}, i}$ as the maximal force that the motor can sustain in motor state $i$.

The most weakly bound state of kinesin is provided by the (DD)-state. Thus, in order to reduce the number of parameters, one may assume that the motor unbinds primarily from the (DD) state with $i=7$ as shown in Fig. 4(b). Furthermore, for small values of $F$, the unbinding rate $\omega_{\text {off }}$ of kinesin is of the order of $1 / \mathrm{s}$ whereas the zero-force transition rates between the different bound states $i=1,2, \ldots, 7$ are of the order of or larger than $1 /(10 \mathrm{~ms})$. This separation of time scales implies that the kinesin motor has essentially attained the steady state of the 7-state network before it starts to undergo transitions from the bound 

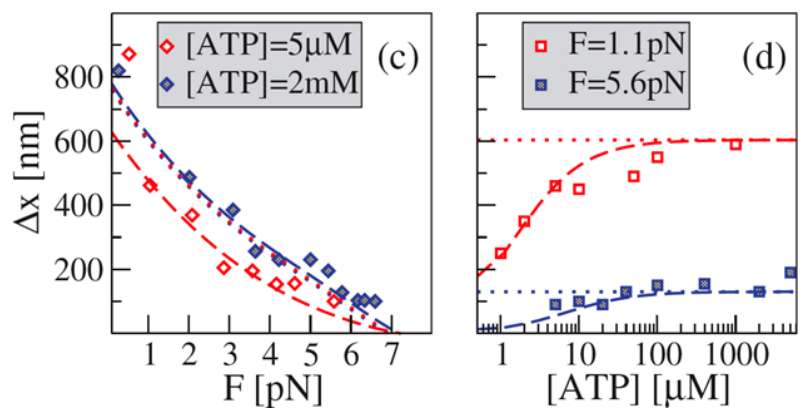

Fig. 9 Average run length (or walking distance) $\langle\Delta x\rangle$ of kinesin as a function (a) of load force $F$ and (b) of ATP concentration. With increasing $F$, the run length first decays by an exponential factor that is governed by the detachment force $F_{\mathrm{d}}$ and then vanishes at the stall force $F=F_{\mathrm{S}}$, compare Fig. 6 . The experimental data are from [18], the broken lines represent the behavior as calculated for the network in Fig. 4(b) with $[\mathrm{ADP}]=[\mathrm{P}]=0.5 \mu \mathrm{M}[3]$. These latter calculations lead to the detachment force $F_{\mathrm{d}, 7} \simeq 5 \mathrm{pN}$ [3] while a simple exponential fit to the data implies $F_{\mathrm{d}} \simeq 3 \mathrm{pN}$ as explained in the text

state $i=7$ to the unbound state $i=0$ provided the load force $F$ is sufficiently small. The unbinding rate of the motor can then be estimated by [3]

$$
\omega_{\text {off }} \simeq P_{7}^{\text {st }} \omega_{70}=P_{7}^{\text {st }} \kappa_{70} \exp \left(F / F_{\mathrm{d}, 7}\right)=P_{7}^{\text {st }} \kappa_{70} \exp \left(\chi_{70} \bar{F}\right) .
$$

This unbinding rate depends on the load force $F$ both via the explicit exponential factor and via the occupation probability $P_{7}^{\text {st }}=P_{7}^{\text {st }}(F)$. Comparison with relation (29) then leads to the zero-force unbinding rate

$$
\kappa_{\mathrm{off}} \simeq P_{7}^{\mathrm{st}}(0) \kappa_{70} .
$$

Likewise, the overall detachment force $F_{\mathrm{d}}$ in (29) will, in general, differ from $F_{\mathrm{d}, 7}$ because of the force dependence of $P_{7}^{\text {st }}$.

The average run time $\langle\Delta t\rangle$ should decrease monotonically with increasing $F$ and vanishes in the limit of large $F$. In contrast, the average run length $\langle\Delta x\rangle=v\langle\Delta t\rangle$ vanishes already at the stall force $F=F_{\mathrm{s}}$, at which the motor velocity $v$ changes sign, compare Fig. 6.

For kinesin, the $F$-dependence of the run length is displayed in Fig. 9(a) where the experimental data of Ref. [18] are compared with calculations based on the chemomechanical network in Fig. 4(b) as described in Ref. [3]. The latter calculations lead to the rate constant $\kappa_{70} \simeq 3 / \mathrm{s}$ and to the parameter $\chi_{70}=0.1$, which implies the detachment force $F_{\mathrm{d}, 7}=k_{B} T / \ell \chi_{70} \simeq 5 \mathrm{pN}$ at room temperature. On the other hand, if the data in Fig. 9(a) are directly fitted as $\langle\Delta x\rangle \sim \exp \left(-F / F_{\mathrm{d}}\right)$, one obtains the overall detachment force $F_{\mathrm{d}} \simeq 3 \mathrm{pN}$ as deduced in Ref. [18]. Another set of data that is well described by the 7-state network model is the dependence of the run length on the ATP concentration, see Fig. 9(b).

For the 7-state network in Fig. 4(b), the zero-force unbinding rate $\kappa_{\text {off }}$ as given by (33) is proportional to the steady state probability $P_{7}^{\text {st }}$ that the motor occupies the (DD) state. Inspection of Fig. 4(b) shows that this occupation probability decreases as one increases the ATP concentration since one then decreases the flux from state (DE) with $i=1$ to state (DD) with $i=7$ relative to the flux from state (DE) to state (DT) with $i=2$. For the same reason, the probability $P_{7}^{\text {st }}$ also decreases if one decreases the ADP concentration. Thus, the 7-state model in Fig. 4(b) predicts that the zero-force unbinding rate $\kappa_{\text {off }}$ depends on the nucleotide concentrations. 
In order to check the assumed separation of time scales, one may consider the unbinding process as another 'absorption' process with only one absorbing state given by $i=0$. If the motor starts in the initial state $i=a$, the probability distribution for the process to exhibit the run time $s \equiv \Delta t$ is given by

$$
\rho_{a \mid 0}^{\mathrm{ab}}(s)=\frac{\partial}{\partial s} P_{a 0}(s)=P_{a 7}(s) \omega_{70},
$$

compare relation (25). Now, let $P_{a}^{\text {in }}$ be the probability that the motor is initially in state $i=a$. The probability distribution for the run time $s$ is then given by

$$
\rho_{0}^{\mathrm{ab}}(s)=\sum_{a \neq 0} P_{a}^{\mathrm{in}} P_{a 7}(s) \omega_{70},
$$

and the average run time $\langle\Delta t\rangle$ is equal to the first moment of this distribution.

\section{Summary and Outlook}

In this article, we have reviewed recent work on the chemomechanical coupling of molecular motors, which determines the stochastic energy conversion by these motors. This coupling is described by chemomechanical networks as shown in Figs. 2 and 4 [5, 6]. The network models for kinesin in Fig. 4 are able to describe all experimental data as obtained from single motor measurements [3]. In particular, they lead to a quantitative description of the motor velocity and run length as functions of load force, see Fig. 6 and Fig. 9, which determine the motor's stall and detachment force, respectively.

For the 6-state model in Fig. 4(c), the stall force can be calculated explicitly as a function of chemical energy input and, thus, of ATP concentration, see relation (21). Another interesting set of quantities that has been calculated for this 6-state model is provided by the dwell time distributions for the mechanical steps as discussed in Sect. 8. These dwell times are difficult to measure with a high temporal resolution but all available data are again well described by this model without introducing any additional fit parameters [4].

Because of thermal noise, molecular motors unbind from the filaments after a finite run length. The average run length of a single motor depends on the load force $F$ and the nucleotide concentrations, as shown for kinesin in Fig. 9. For small $F$ and large ATP concentration, the average run length of this motor is about $800 \mathrm{~nm}$ corresponding to about 100 forward steps. When a single kinesin pulls a cargo particle or bead, its velocity and run length is hardly affected even if the particle has a size of many micrometers because the hydrodynamic friction produces only a relatively small force on the motor.

The run length of a single motor is, however, quite small compared to the long distances - centimeters or even meters-over which vesicles, filaments, and organelles are transported by these motors in cells and axons. One rather effective way to increase the run length is via cooperative transport by small teams of motors [41-43]. In addition, cargo particles often exhibit complex transport patterns of bi-directional transport which can be explained in terms of a stochastic tug-of-war between two antagonistic motor teams attached to the same cargo [40, 44, 45]. Finally, the simultaneous transport of many cargo particles leads to heavy traffic within eukaryotic cells. This traffic can be modelled using extensions of asymmetric simple exclusion processes and leads to a variety of cooperative phenomena: build-up of traffic jams, active pattern formation with spatially nonuniform density and flux patterns, and traffic phase transitions. These latter phenomena are reviewed in $[39,46,47]$. 
Acknowledgements This work has been supported by STREP project No. NMP4-CT-2004-516989 within the EC sixth Framework Program.

Open Access This article is distributed under the terms of the Creative Commons Attribution Noncommercial License which permits any noncommercial use, distribution, and reproduction in any medium, provided the original author(s) and source are credited.

\section{Appendix: Local and Nonlocal Balance Relations}

In this appendix, we briefly review the local and nonlocal balance relations obtained in Refs. $[5,6]$. In addition, we generalize the local relations as derived in [6] by removing an implicit and unnecessary assumption.

As emphasized in Ref. [6], each motor state $i$ represents an ensemble of substates $\left(i, k_{i}\right)$ that is thermally equilibrated at temperature $T$. If the energy of substate $\left(i, k_{i}\right)$ is denoted by $E\left(i, k_{i}\right)$, the conditional probability $P\left(k_{i} \mid i\right)$ to find the motor molecule in substate $\left(i, k_{i}\right)$, when we know that it is in state $i$, has the form

$$
P\left(k_{i} \mid i\right)=\frac{1}{Z_{i}} e^{-E\left(i, k_{i}\right) / k_{B} T} \quad \text { with } Z_{i} \equiv \sum_{k_{i}} e^{-E\left(i, k_{i}\right) / k_{B} T} .
$$

When the motor is in state $i$, it has the internal energy

$$
U_{i} \equiv\left\langle E\left(i, k_{i}\right)\right\rangle=\sum_{k_{i}} P\left(k_{i} \mid i\right) E\left(i, k_{i}\right),
$$

the Helmholtz free energy

$$
H_{i}=-k_{B} T \ln \left(Z_{i}\right),
$$

and the entropy

$$
S_{i}=\left(U_{i}-H_{i}\right) / T .
$$

When the motor undergoes a transition $|i j\rangle$ from substate $\left(i, k_{i}\right)$ to substate $\left(j, k_{j}\right)$, the corresponding energy change $E\left(j, k_{j}\right)-E\left(i, k_{i}\right)$ will, in general, fluctuate. The average value of this quantity determines the internal energy change

$$
U_{j}-U_{i}=\left\langle E\left(j, k_{j}\right)-E\left(i, k_{i}\right)\right\rangle=\sum_{k_{j}} P\left(k_{j} \mid j\right) E\left(j, k_{j}\right)-\sum_{k_{i}} P\left(k_{i} \mid i\right) E\left(i, k_{i}\right) .
$$

As discussed in Sect. 5, conservation of energy implies (7) as given by

$$
U_{j}-U_{i}=E_{\mathrm{ch}, i j}-W_{\mathrm{me}, i j}-Q_{i j} .
$$

Thus, the three quantities on the right hand side of this equation should also be regarded as average quantities, which are obtained by averaging over many transitions $|i j\rangle$.

The local balance relations are obtained by expressing the released heat $Q_{i j}$ in terms of the transition rates $\omega_{i j}$ and $\omega_{j i}$ for the transitions $|i j\rangle$ and $|j i\rangle$. In order to do this, one can consider a continuous-time Markov process on the network of motor states. The probabilities $P_{i}$ to find the motor in state $i$ are then governed by the master equation

$$
\frac{d}{d t} P_{i}=-\sum_{j}\left(P_{i} \omega_{i j}-P_{j} \omega_{j i}\right) \equiv-\sum_{j} \Delta J_{i j},
$$

where the last equality defines the local excess fluxes $\Delta J_{i j}$. 
In the steady state with $P_{i}=P_{i}^{\text {st }}$, these local excess fluxes satisfy the flux balance relations

$$
\sum_{j} \Delta J_{i j}^{\mathrm{st}}=\sum_{j}\left(P_{i}^{\mathrm{st}} \omega_{i j}-P_{j}^{\mathrm{st}} \omega_{j i}\right)=0
$$

and the entropy production rate has the general form [33, 48-50]

$$
\sigma_{\mathrm{pr}}^{\mathrm{st}}=\frac{1}{2} \sum_{i} \sum_{j}^{\prime} \Delta J_{i j}^{\mathrm{st}} k_{B} \ln \left(\omega_{i j} / \omega_{j i}\right),
$$

where the two summation indices $i$ and $j$ run over all motor states of the network and the prime indicates that there are no terms with $j=i$.

As explained in [6], the expression (43) for the entropy production rate $\sigma_{\mathrm{pr}}^{\text {st }}$ can be rewritten in two ways. First, one may replace the double summation over $i$ and $j$ by a summation over all di-edges $|i j\rangle$. Second, one may also derive a decomposition of the entropy production rate in terms of dicycle fluxes [5]. The first decomposition in terms of di-edges leads to

$$
\sigma_{\mathrm{pr}}^{\mathrm{st}}=\sum_{|i j\rangle} P_{i}^{\mathrm{st}} \omega_{i j} k_{B} \ln \left(\omega_{i j} / \omega_{j i}\right) \equiv \sum_{|i j\rangle} P_{i}^{\mathrm{st}} \omega_{i j} \Delta S_{i j}
$$

Since $P_{i}^{\text {st }} \omega_{i j}$ is the frequency with which the motor undergoes the transition $|i j\rangle$, the quantity $\Delta S_{i j}=k_{B} \ln \left(\omega_{i j} / \omega_{j i}\right)$ represents the average entropy produced during the transition $|i j\rangle$ [30]. In Ref. [6], we then identified $T \Delta S_{i j}$ with the heat $Q_{i j}$ released by the motor into the heat reservoir. The latter identification is, however, restricted to motor states $i$ and $j$ with $S_{i}=S_{j}$ as explained in the following.

In general, the two states $i$ and $j$ will differ in their entropies $S_{i}$ and $S_{j}$ as defined in (39). The average entropy $\Delta S_{i j}$ produced during the transition $|i j\rangle$ is then postulated to satisfy the more general relation

$$
\Delta S_{i j}=k_{B} \ln \left(\omega_{i j} / \omega_{j i}\right)=S_{j}-S_{i}+Q_{i j} / T
$$

where $S_{j}-S_{i}$ and $Q_{i j} / T$ represent the entropy change of the motor molecule and of the heat bath, respectively. An analogous decomposition of the entropy change is always valid for isothermal processes of macroscopic systems, see, e.g., [51].

If we substitute the expression (45) into the entropy production rate $\sigma_{\mathrm{pr}}^{\text {st }}$ as given by (43), we obtain

$$
\sigma_{\mathrm{pr}}^{\mathrm{st}}=\frac{1}{2} \sum_{i} \sum_{j}^{\prime} \Delta J_{i j}^{\mathrm{st}} Q_{i j} / T,
$$

since the terms proportional to $S_{j}-S_{i}$ cancel out from this expression. In fact, one has

$$
\frac{1}{2} \sum_{i} \sum_{j}^{\prime} \Delta J_{i j}^{\mathrm{st}}\left(A_{j}-A_{i}\right)=0
$$

for any state variable $A_{i}$ as follows from the flux balance relations (42) together with $\Delta J_{j i}=$ $-\Delta J_{i j}$. Thus, the form (43) of the entropy production rate $\sigma_{\mathrm{pr}}^{\text {st }}$ is consistent with any relation of the form $\Delta S_{i j}=k_{B} \ln \left(\omega_{i j} / \omega_{j i}\right)=A_{j}-A_{i}+Q_{i j} / T$ but does not allow us to actually determine the state variable $A_{i}$.

In order to show that the state variable $A_{i}$ is indeed equal to the entropy $S_{i}$ of the motor state $i$ as postulated in (45), we now consider the constrained equilibrium of a subsystem 
consisting of only two states $a$ and $b$ in thermal equilibrium with a heat reservoir as in [6], and first assume that this two-state system is not coupled to any other reservoir. Energy conservation during a transition $|a b\rangle$ from state $a$ to state $b$ is now described by

$$
U_{b}-U_{a}=-Q_{a b}
$$

which represents a special case of local energy conservation as described by (7). If the substates of the two states $a$ and $b$ are denoted by $\left(a, k_{a}\right)$ and $\left(b, k_{b}\right)$ and have energies $E\left(a, k_{a}\right)$ and $E\left(b, k_{b}\right)$, the canonical partition functions of these two subensembles are given by

$$
Z_{a}=\sum_{k_{a}} e^{-E\left(a, k_{a}\right) / k_{B} T}=e^{-\left(U_{a}-T S_{a}\right) / k_{B} T}
$$

and

$$
Z_{a}=\sum_{k_{b}} e^{-E\left(b, k_{b}\right) / k_{B} T}=e^{-\left(U_{b}-T S_{b}\right) / k_{B} T} .
$$

Furthermore, the probabilities $P_{a}$ and $P_{b}$ to find the two-state system in the states $a$ and $b$ can be expressed as

$$
P_{a}=\frac{Z_{a}}{Z_{a}+Z_{b}} \quad \text { and } \quad P_{b}=\frac{Z_{b}}{Z_{a}+Z_{b}}
$$

If these relations are combined with the detailed balance condition $P_{a} \omega_{a b}=P_{b} \omega_{b a}$, one obtains

$$
k_{B} \ln \left(\omega_{a b} / \omega_{b a}\right)=S_{b}-S_{a}-\left(U_{b}-U_{a}\right) / T=S_{b}-S_{a}+Q_{a b} / T,
$$

where the last equality follows from (48). Therefore, the postulated relation (45) is indeed valid for a two-state system coupled to a heat reservoir. It is not difficult to repeat this calculation for a two-state system in constrained equilibrium coupled both to a heat reservoir and to additional reservoirs such as a particle exchange reservoir and/or a work reservoir. In all cases, one recovers the relation (45).

If the decomposition (45) of the produced entropy is combined with the relation (7) for local energy conservation, one arrives at the local balance relation

$$
H_{j}-H_{i}=U_{j}-U_{i}-T\left(S_{j}-S_{i}\right)=E_{\mathrm{ch}, i j}-W_{\mathrm{me}, i j}-k_{B} T \ln \left(\omega_{i j} / \omega_{j i}\right),
$$

for the free energies $H_{i}$ which generalizes relation (6.15) in Ref. [6] to the case $S_{i} \neq S_{j}$. In fact, all relations in Ref. [6] that follow after equation (6.15) of this article and involve the internal energies $U_{i}$ can now be generalized to the case $S_{j} \neq S_{i}$ by simply replacing the internal energies $U_{i}$ by the free energies $H_{i}$.

If one considers the sum $\sum_{|i j\rangle}\left(H_{j}-H_{i}\right)$ over all transitions $|i j\rangle$ of a certain dicyle $\mathcal{C}_{v}^{d}$, all free energy differences $H_{j}-H_{i}$ cancel out, and the local relation (53) then leads to the nonlocal balance relation

$$
Q\left(\mathcal{C}_{v}^{d}\right)=k_{B} T \ln \left(\prod_{|i j\rangle}^{v, d} \omega_{i j} / \prod_{|j i\rangle}^{v,-d} \omega_{j i}\right)=E_{\mathrm{ch}}\left(\mathcal{C}_{v}^{d}\right)-W_{\mathrm{me}}\left(\mathcal{C}_{v}^{d}\right)
$$

for the average heat released during the completion of dicycle $\mathcal{C}_{v}^{d}$. This relation is identical with equation (14) in Sect. 5 and was previously derived in [5, 6]. In fact, apart from equation (7) for the internal energy differences $U_{j}-U_{i}$, all relations in Sects. 5-7 are expressed in terms of dicycle quantities and, thus, do not depend on the state variables $U_{i}, H_{i}$, or $S_{i}$. 


\section{Glossary of Abbreviations and Symbols}

ADP adenosin diphosphate.

[ADP] molar concentration of ADP.

ADP $/ \mathrm{P}$ product of ATP cleavage, abbreviated as $\Theta$.

ATP adenosin triphosphate.

[ATP] molar concentration of ATP.

$\mathcal{B}$ backward cycle of single motor.

$\mathcal{C}_{v} \quad$ cycle of network graph labeled by index $v$.

$\mathcal{C}_{v}^{d} \quad$ directed cycle or dicycle of network graph with direction $d$.

$d \quad$ direction of dicycle with $d= \pm$.

$\mathcal{D} \quad$ dissipative slip cycle of single motor.

$\Delta J_{i j} \quad$ local excess flux of transition $|i j\rangle$ with $\Delta J_{i j}=J_{i j}-J_{j i}$.

$\Delta J\left(\mathcal{C}_{v}^{d}\right) \quad$ dicycle excess flux as in (15).

$\Delta J^{\mathrm{st}}\left(\mathcal{C}_{v}^{d}\right)$ dicycle excess flux in steady state.

$\Delta \mu \quad$ chemical energy input from a single ATP hydrolysis as in (4).

$\Delta \bar{\mu} \quad$ reduced chemical energy input with $\Delta \bar{\mu}=\Delta \mu / k_{B} T$.

$\Delta t \quad$ run time of motor at filament.

$\langle\Delta t\rangle \quad$ average run time of motor.

$\Delta x \quad$ run length (or walking distance) of motor along filament.

$\langle\Delta x\rangle \quad$ average run length of motor.

$E_{\text {ch }} \quad$ chemical energy input to the motor as in (1) and (4).

$E_{\mathrm{ch}, i j}$

$E_{\mathrm{ch}}\left(\mathcal{C}_{v}^{d}\right)$ chemical energy input to the motor during transition $|i j\rangle$.

$F \quad$ load force acting on the motor; $F>0$ for resisting load.

$F_{\mathrm{d}} \quad$ detachment force of single motor.

$F_{\mathrm{d}, i} \quad$ detachment force for single motor in state $i$.

$F_{\mathrm{s}} \quad$ stall force of single motor.

$\mathcal{F} \quad$ forward cycle of single motor.

$H_{i} \quad$ Helmholtz free energy of motor state $i$ as defined in (38).

$i, j \quad$ discrete states of a single motor bound to a filament.

$|i j\rangle \quad$ transition or directed edge (di-edge) from state $i$ to state $j$.

$\langle i j\rangle \quad$ edge between states $i$ and $j$.

$J_{i j} \quad$ probability flux from motor state $i$ to state $j$ with $J_{i j}=P_{i} \omega_{i j}$.

$J\left(\mathcal{C}_{v}^{d}\right) \quad$ dicycle flux as in (16).

$J^{\text {st }}\left(\mathcal{C}_{v}^{d}\right) \quad$ dicycle flux in steady state.

$\kappa_{i 0}$

$\kappa_{\text {off }}$

$k_{B}$

$K^{\mathrm{eq}}$

$\ell$

$M_{\mathrm{bs}}$

$M_{\mathrm{cd}}$

$\mu$

$\mu(X) \quad$ chemical potential for chemical species $X$ as in (3).

$v$

$\omega_{i j} \quad$ transition rate for transition from motor state $i$ to state $j$.

$\mathrm{P} \quad$ inorganic phosphate.

$P_{i} \quad$ probability that motor is in internal state $i$. 


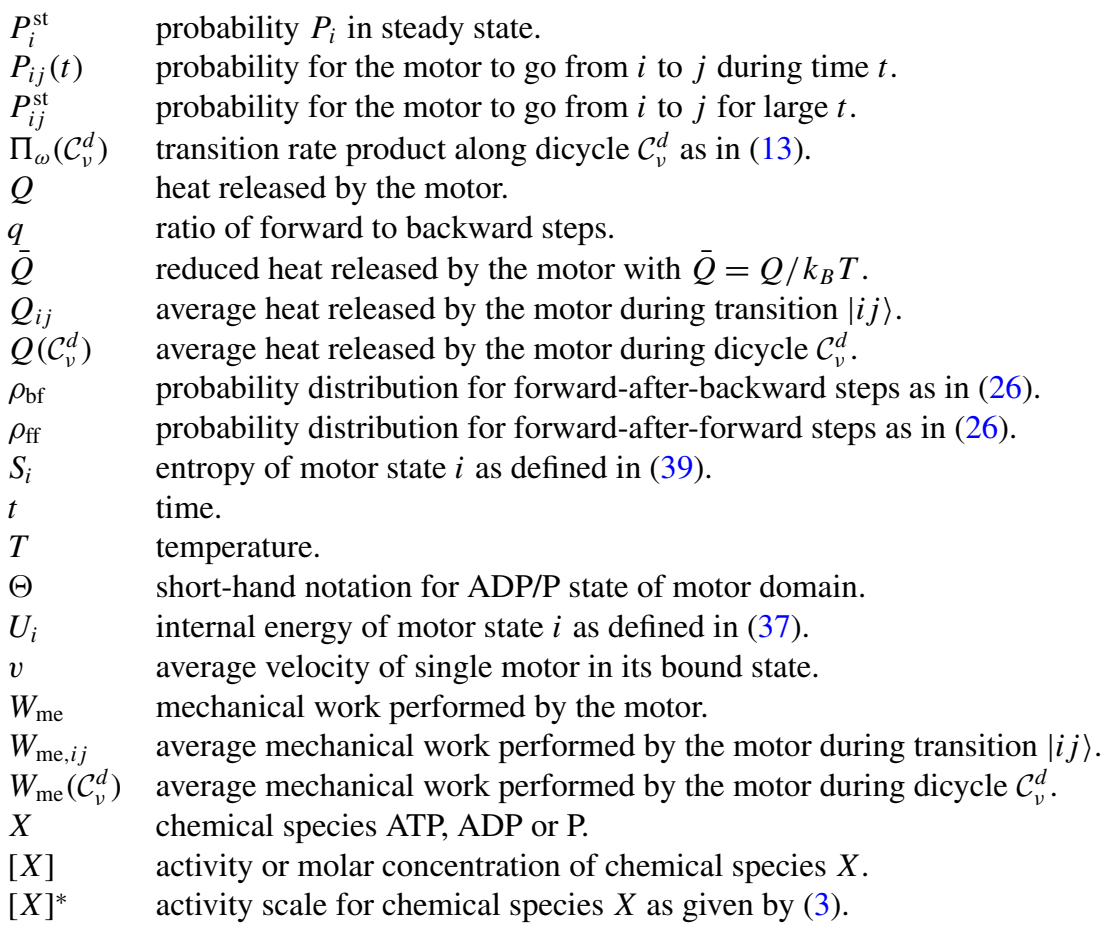

Note Added in Proof The compact network in Fig. 4(a) corresponds to the local "view" of the motor and treats chemical and mechanical transitions on the same footing. The chemomechanical cycles contained in this compact network are also present in the network in Fig. 3 provided this latter network is supplemented by periodic boundary conditions. Note that cycles in a discrete state space play an analogous role to holes in a continuous, multiply-connected space.

\section{References}

1. Alberts, B., Bray, D., Johnson, A., Lewis, J., Raff, M., Roberts, K., Walter, P.: Essential Cell Biology: An Introduction to the Molecular Biology of the Cell. Garland, New York (1998)

2. Schliwa, M. (ed.): Molecular Motors. Wiley-VCH, Weinheim (2003)

3. Liepelt, S., Lipowsky, R.: Kinesin's network of chemomechanical motor cycles. Phys. Rev. Lett. 98, 258102 (2007)

4. Valleriani, A., Liepelt, S., Lipowsky, R.: Dwell time distributions for kinesin's mechanical steps. EPL 82, 28011 (2008)

5. Liepelt, S., Lipowsky, R.: Steady-state balance conditions for molecular motor cycles and stochastic nonequilibrium processes. EPL 77, 50002 (2007)

6. Lipowsky, R., Liepelt, S.: Chemomechanical coupling of molecular motors: thermodynamics, network representations, and balance conditions. J. Stat. Phys. 130, 39-67 (2008)

7. Svoboda, K., Schmidt, Ch.F., Schnapp, B.J., Block, St.M.: Direct observation of kinesin stepping by optical trapping interferometry. Nature 365, 721-727 (1993)

8. Yildiz, A., Tomishige, M., Vale, R.D., Selvin, P.R.: Kinesin walks hand-over-hand. Science 303, 676-678 (2004)

9. Carter, N.J., Cross, R.A.: Mechanics of the kinesin step. Nature 435, 308-312 (2005)

10. Schnitzer, M.J., Block, St.M.: Kinesin hydrolyses one ATP per 8-nm step. Nature 388, 386-390 (1997)

11. Hackney, D.D.: The tethered motor domain of a kinesin-microtubule complex catalyzes reversible synthesis of bound ATP. PNAS 102, 18338-18343 (2005)

12. Gilbert, S.P., Moyer, M.L., Johnson, K.A.: Alternating site mechanism of the kinesin ATPase. Biochemistry 37, 792-799 (1998) 
13. Romberg, L., Vale, R.D.: Chemomechanical cycle of kinesin differs from that of myosin. Nature 361, 168-170 (1993)

14. Crevel, I., Lockhart, A., Cross, R.A.: Weak and strong states of kinesin and ncd. J. Mol. Biol. 257, 66-76 (1996)

15. Guydosh, N.R., Block, S.M.: Backsteps induced by nucleotide analogs suggest the front head of kinesin is gated by strain. Proc. Nat. Acad. Sci. USA 103, 8054-8059 (2006)

16. Visscher, K., Schnitzer, M.J., Block, S.M.: Single kinesin molecules studied with a molecular force clamp. Nature 400, 184-189 (1999)

17. Nishiyama, M., Higuchi, H., Yanagida, T.: Chemomechanical coupling of the forward and backward steps of single kinesin molecules. Nat. Cell Biol. 4, 790-797 (2002)

18. Schnitzer, M.J., Visscher, K., Block, S.M.: Force production by single kinesin motors. Nat. Cell Biol. 2, 718-723 (2000)

19. Schief, W.R., Clark, R.H., Crevenna, A.H., Howard, J.: Inhibition of kinesin motility by ADP and phosphate supports a hand-over-hand mechanism. PNAS 101, 1183-1188 (2004)

20. Block, S.M., Goldstein, L.S.B., Schnapp, B.J.: Bead movement by single kinesin molecules studied with optical tweezers. Nature 348, 348-352 (1990)

21. Hill, T.L.: Free Energy Transduction and Biochemical Cycle Kinetics, 1st edn. Springer, New York (1989)

22. Yildiz, A., Forkey, J.N., McKinney, S.A., Ha, T., Goldmann, Y.E., Selvin, P.R.: Myosin V walks handover-hand: single fluorophore imaging with 1.5-nm localization. Science 300, 2061-2065 (2003)

23. Veigel, C., Wang, F., Bartoo, M.L., Sellers, J.R., Molloy, J.E.: The gated gait of the processive molecular motor, myosin V. Nat. Cell Biol. 4, 59-65 (2002)

24. Cappello, G., Pierobon, P., Symonds, C., Busoni, L., Gebhardt, J., Rief, M., Prost, J.: Myosin V stepping mechanism. Proc. Nat. Acad. Sci. USA 104, 15328-15333 (2007)

25. Lipowsky, R.: Universal aspects of the chemo-mechanical coupling for molecular motors. Phys. Rev. Lett. 85, 4401-4404 (2000)

26. Lipowsky, R., Jaster, N.: Molecular motor cycles: from ratchets to networks. J. Stat. Phys. 110, 11411167 (2003)

27. Schmiedl, T., Seifert, U.: Stochastic thermodynamics of chemical reaction networks. Phys. Rev. E 126, 044101 (2007)

28. Andrieux, D., Gaspard, P.: Temporal disorder and fluctuation theorem in chemical reactions. Phys. Rev. E 77, 031137 (2008)

29. Alberty, R.A.: Thermodynamics of the hydrolysis of ATP as a function of temperature, $\mathrm{pH}, \mathrm{pMg}$, and ionic strength. J. Phys. Chem. B 107, 12324-12330 (2003)

30. Seifert, U.: Fluctuation theorem for a single enzym or molecular motor. Europhys. Lett. 70, 36-41 (2005)

31. Evans, D.J., Cohen, E.G.D., Morris, G.P.: Probability of second law violations in shearing steady states. Phys. Rev. Lett. 71, 2401-2404 (1993)

32. Crooks, G.E.: Entropy production fluctuation theorem and the nonequilibrium work relation for free energy differences. Phys. Rev. E 60, 2721-2726 (1999)

33. Lebowitz, J.L., Spohn, H.: A Gallavotti-Cohen-type symmetry in the large deviation functional for stochastic dynamics. J. Stat. Phys. 95, 333-365 (1999)

34. Jarzynski, C.: Hamiltonian derivation of a detailed fluctuation theorem. J. Stat. Phys. 98, 77-102 (2000)

35. Liepelt, S., Lipowsky, R.: Operation modes of the molecular motor kinesin. Phys. Rev. E 79, 011917 (2009)

36. King, St.J., Schroer, T.A.: Dynactin increases the processivity of the cytoplasmic dynein motor. Nat. Cell Biol. 2, 20-24 (2000)

37. Mehta, A.D., Rock, R.S., Rief, M., Spudich, J.A., Mooseker, M.S., Cheney, R.E.: Myosin-V is a processive actin-based motor. Nature 400, 590-593 (1999)

38. Ökten, Z., Churchman, L.S., Rock, R.S., Spudich, J.A.: Myosin VI walks hand-over-hand along actin. Nat. Struct. Mol. Biol. 11, 884-887 (2004)

39. Lipowsky, R., Chai, Y., Klumpp, S., Liepelt, S., Müller, M.J.I.: Molecular motor traffic: from biological nanomachines to macroscopic transport. Physica A 372, 34-51 (2006)

40. Müller, M.J.I., Klumpp, S., Lipowsky, R.: Tug-of-war as a cooperative mechanism for bidirectional cargo transport by molecular motors. Proc. Nat. Acad. Sci. USA 105, 4609-4614 (2008)

41. Klumpp, S., Lipowsky, R.: Cooperative cargo transport by several molecular motors. Proc. Nat. Acad. Sci. USA 102, 17284-17289 (2005)

42. Vershinin, M., Carter, B.C., Razafsky, D.S., King, S.J., Gross, S.P.: Multiple-motor based transport and its regulation by Tau. Proc. Nat. Acad. Sci. USA 104, 87-92 (2007)

43. Beeg, J., Klumpp, S., Dimova, R., Gracia, R.S., Unger, E., Lipowsky, R.: Transport of beads by several kinesin motors. Biophys. J. 94, 532-541 (2008)

44. Welte, M.A., Gross, S.P.: Molecular motors: a traffic cop within? HFSP J. 2, 178-182 (2008) 
45. Müller, M.J.I., Klumpp, S., Lipowsky, R.: Motility states of molecular motors engaged in a stochastic tug-of-war. J. Stat. Phys. 133, 1059-1081 (2008)

46. Lipowsky, R., Klumpp, S.: Life is motion-multiscale motility of molecular motors. Physica A 352, 53-112 (2005)

47. Lipowsky, R., Beeg, J., Dimova, R., Klumpp, S., Liepelt, S., Müller, M.I.J., Valleriani, A.: Active biosystems: from single motor molecules to cooperative cargo transport. Biophys. Rev. Lett. (2009, in press)

48. Schnakenberg, J.: Network theory of microscopic and macroscopic behavior of master equation systems. Rev. Mod. Phys. 48, 571-585 (1976)

49. Luo, J.L., van den Broeck, C., Nicolis, G.: Stability criteria and fluctuations around non-equilibrium states. Z. Phys. B 56, 165-170 (1984)

50. Maes, Ch., van Wieren, M.H.: A Markov model for kinesin. J. Stat. Phys. 112, 329-355 (2003)

51. Callen, H.B.: Thermodynamics and an Introduction to Thermostatistics, 2nd edn. Wiley, New York (1985) 